Supporting Information

\title{
Composition of Intracellular Protein Corona around Nanoparticles
}

\section{during Internalization}

Chuan Wang, Beibei Chen, Man He, Bin Hu*

Key Laboratory of Analytical Chemistry for Biology and Medicine (Ministry of

Education), Department of Chemistry, Wuhan University, Wuhan 430072, China

*Email: binhu@whu.edu.cn

Figures S1-S13

Tables S1-2, 6-8

Tables S3-5 (see in XLSX file) 


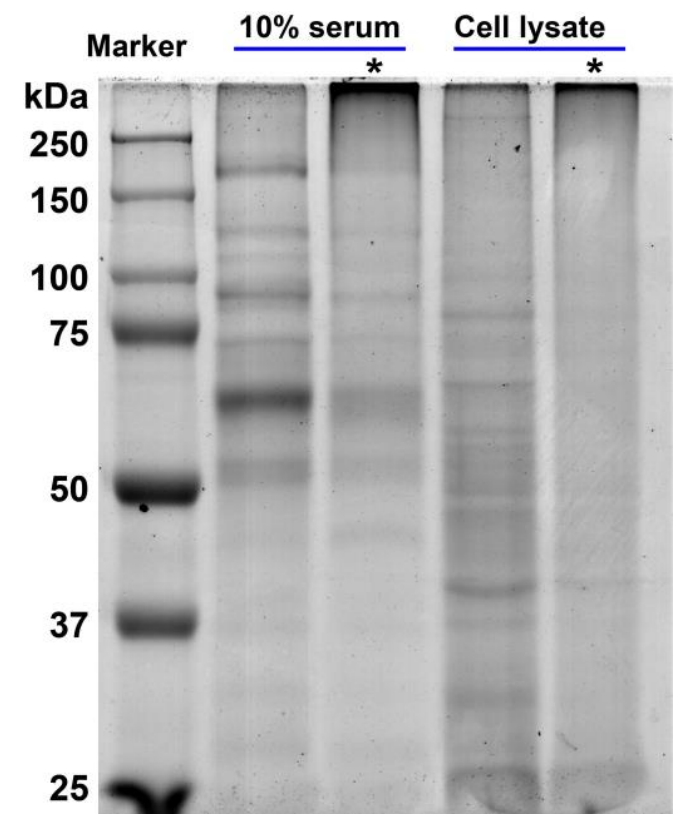

Supplementary Figure S1. SDS-PAGE gel of proteins obtained from cross-linked corona-AuNP complexes formed in $10 \%$ serum at $\mathrm{pH} 4.5$ and cell lysate at $\mathrm{pH} 4.5$. The gel was stained with Coomassie blue. AuNPs were incubated in $10 \%$ serum at $\mathrm{pH} 4.5$ and HepG2 cell lysate at $\mathrm{pH} 4.5$ for $1 \mathrm{~h}$, respectively, and then treated with PFA for 15 min. The resulting AuNP-protein complexes were washed with PBS and collected by centrifugation. PFA-treated samples were boiled at $65{ }^{\circ} \mathrm{C}$ (marked with an asterisk *) and $95{ }^{\circ} \mathrm{C}$ (cross-link reversal) for 10 min prior to SDS-PAGE, respectively. 
(a)

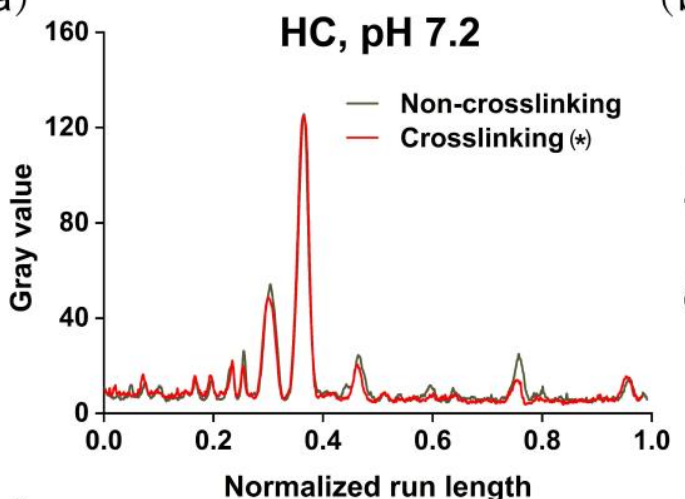

(c)

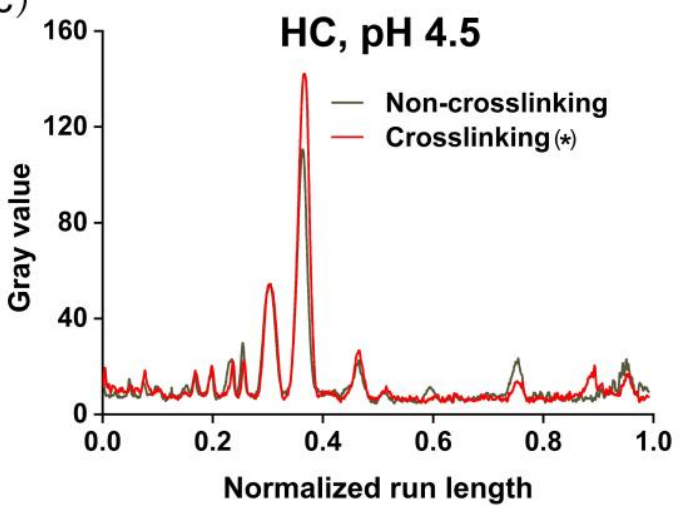

(b)

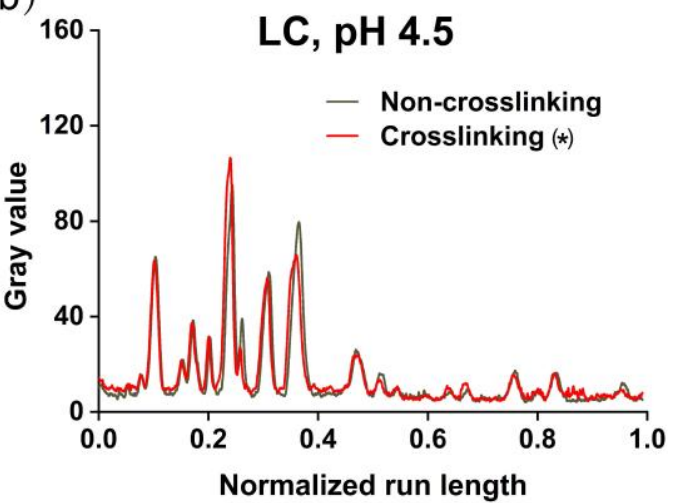

Supplementary Figure S2. Densitometric analysis for gel lanes of non-cross-linking and cross-linking protein coronas in Figure $2 \mathrm{~d}$ of main text, respectively. The run length, shown on the $x$-axis, was normalized to the molecular weight standards and depended on the distance that proteins travelled in the gel. The graphs were generated by ImageJ. 


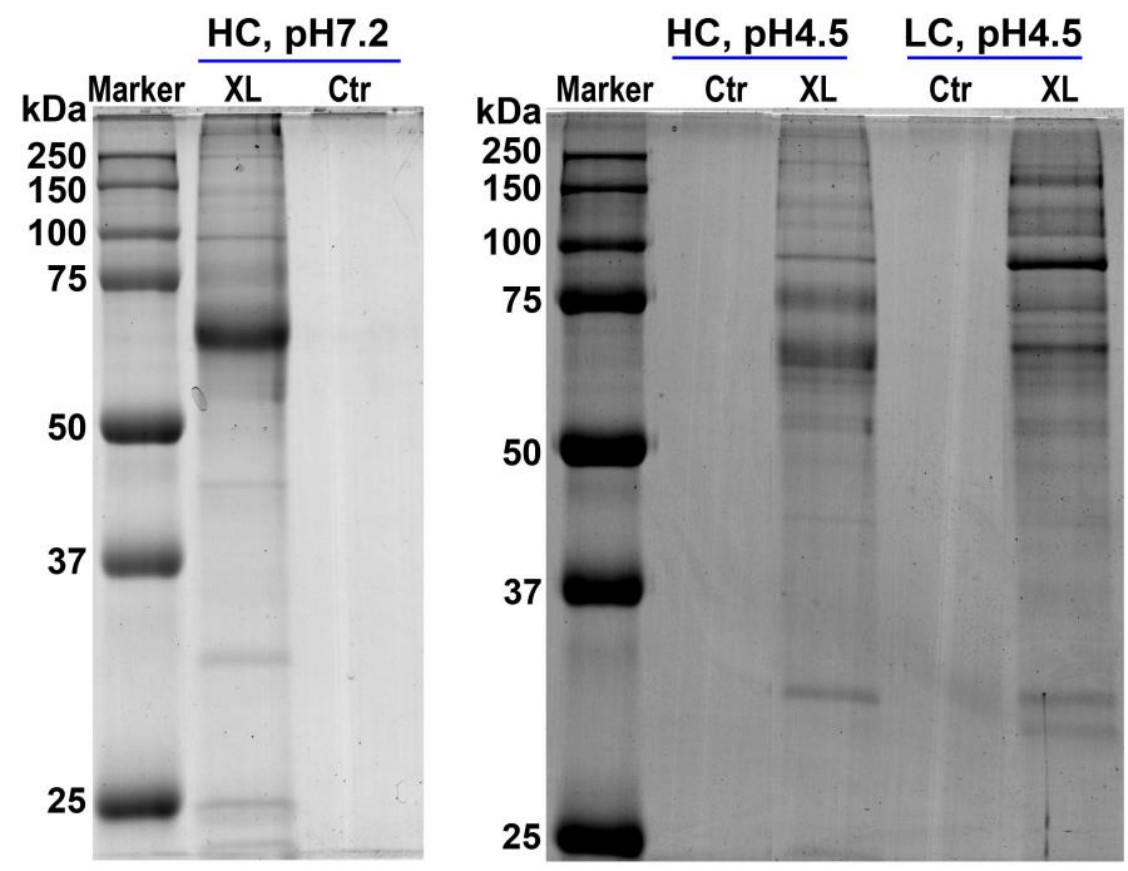

Supplementary Figure S3. Coomassie stained SDS-PAGE gel of the proteins recovered from cross-linked and non-cross-linked AuNP-protein complexes formed in high concentration of serum $(\mathrm{HC}, 80 \%)$ at a physiological $\mathrm{pH} 7.2$ or an acidic $\mathrm{pH} 4.5$, and in low concentration of serum (LC, $10 \%$ ) at an acidic $\mathrm{pH}$ 4.5. Lane XL: the cross-linked sample; Lane Ctr: background control of cross-linking consisting of serum without nanoparticles. 


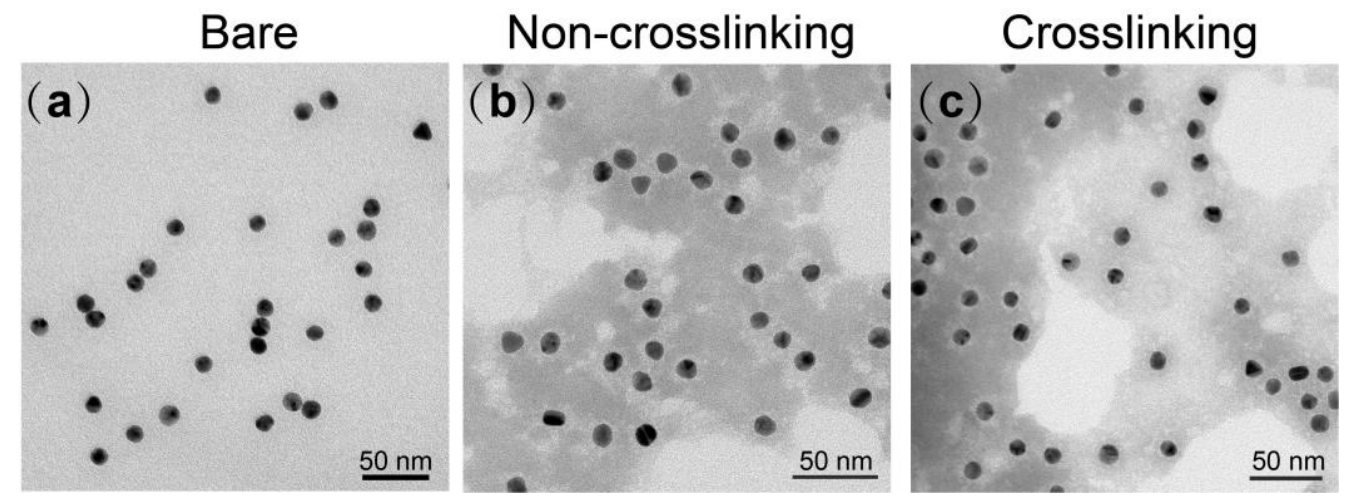

Supplementary Figure S4. TEM images of AuNPs. Negative stain TEM imaging showing well-dispersed AuNPs and similar morphology of AuNP-corona complexes formed in $10 \%$ serum before (a) and after (b) PFA treatment. Bare: AuNPs without protein coating. 


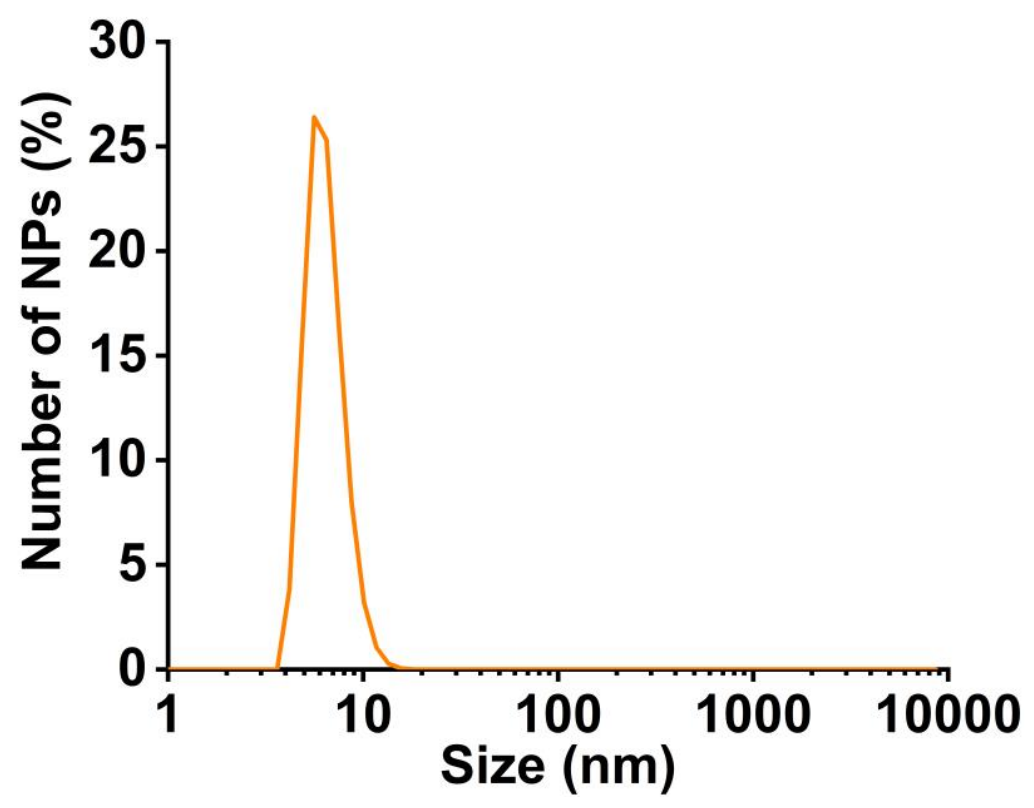

Supplementary Figure S5. DLS analysis of PFA-treated pure serum. This picture shows a sharp peak centered around $7 \mathrm{~nm}$, indicating no prominent protein aggregation. 


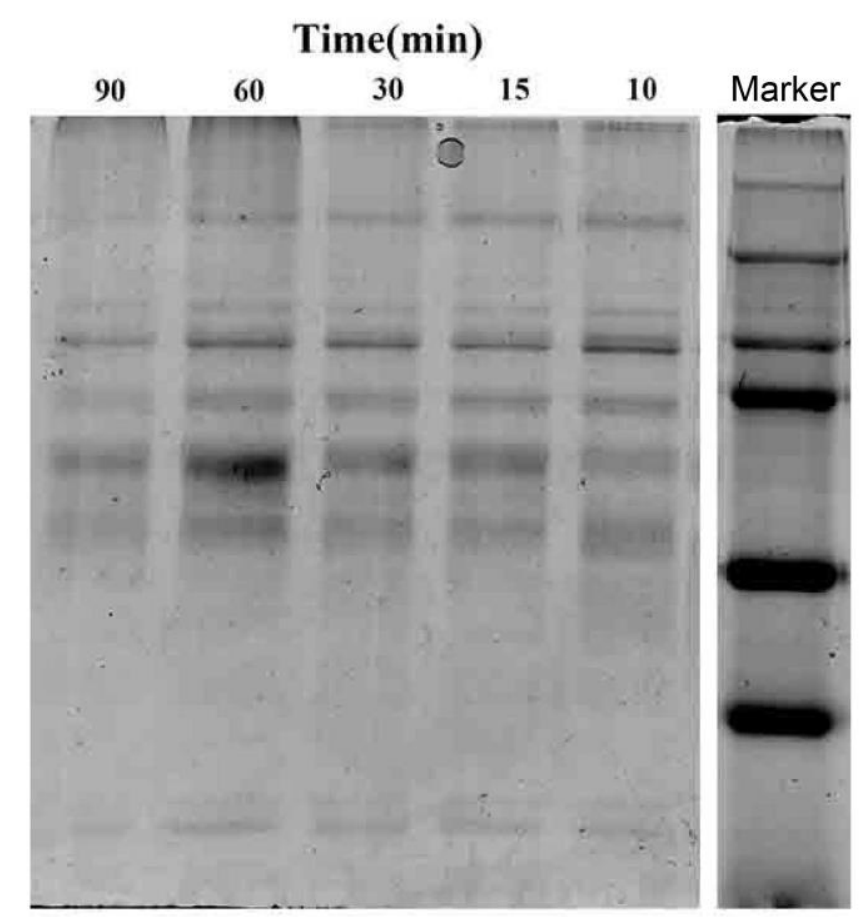

Supplementary Figure S6. Optimizations of cross-linking time. AuNPs were incubated in serum for $1 \mathrm{~h}$, treated with PFA for various times, and then boiled at $95^{\circ} \mathrm{C}$ in SDSPAGE loading buffer. 10-30 min of PFA incubation showed efficient cross-linking reversal for corona proteins, while incubation time of 60 and 90 min resulted in slight band smearing. It implies that the cross-linking reaction was fast, and we chose $15 \mathrm{~min}$ as the optimal condition. 


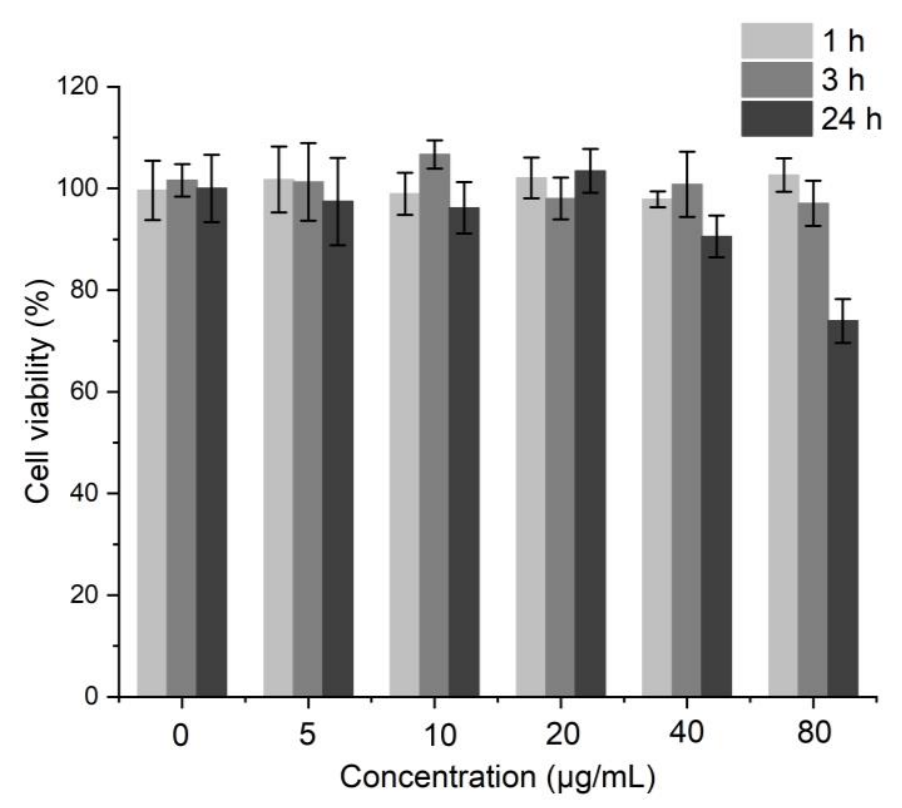

Supplementary Figure S7. Viability of HepG2 cells after 1, 3 and $24 \mathrm{~h}$ of exposure to different concentrations of serum preincubated AuNPs. $1 \times 10^{4} \mathrm{HepG} 2$ cells were seeded per well onto 96-well plates and incubated for $24 \mathrm{~h}$. Then, cell culture medium containing different concentrations of nanoparticles (ranging from 0 to $80 \mu \mathrm{g} / \mathrm{mL}$ ) was introduced separately to each well and further incubated for another 1, 3 and $24 \mathrm{~h}$. The cell viability was assessed by MTT assay as previously described. ${ }^{1}$

\section{Reference}

1. Peng, L.; He, M.; Chen, B. B.; Wu, Q. M.; Zhang, Z. L.; Pang, D. W.; Zhu, Y.; Hu, B. Cellular Uptake, Elimination and Toxicity of CdSe/ZnS Quantum Dots in HepG2 Cells. Biomaterials 2013, 34, 9545-9558. 
(a)

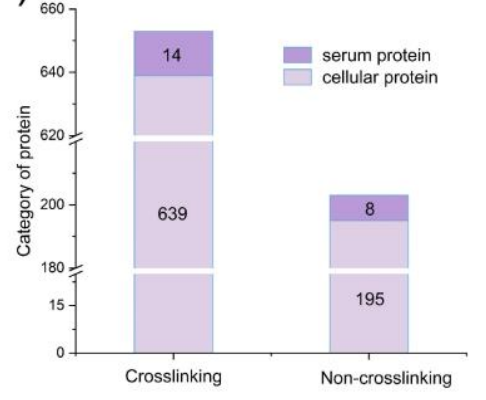

(b)

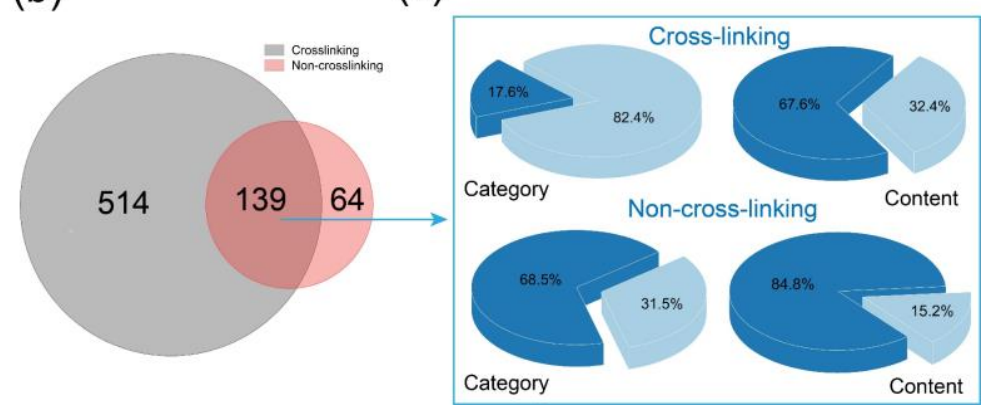

Supplementary Figure S8. Analysis of the protein corona adsorbed onto AuNPs in cells. (a) Classification of identified proteins according to their source (cell or serum). (b) Venn diagrams showed the number of unique proteins identified in the cross-linked and non-cross-linked samples and their respective overlap. (c) The category and content percentage of overlapping proteins (dark blue) in the cross-linked and non-cross-linked corona proteins. A total of 139 proteins overlapped between cross-linked and non-crosslinked corona. The category and contents of these 139 proteins occupied $17.6 \%$ and $67.6 \%$ for cross-linked proteins, and $68.5 \%$ and $84.8 \%$ for non-cross-linked proteins, respectively. High content levels of the overlapping proteins indicated most of the components in these two samples were similar. 


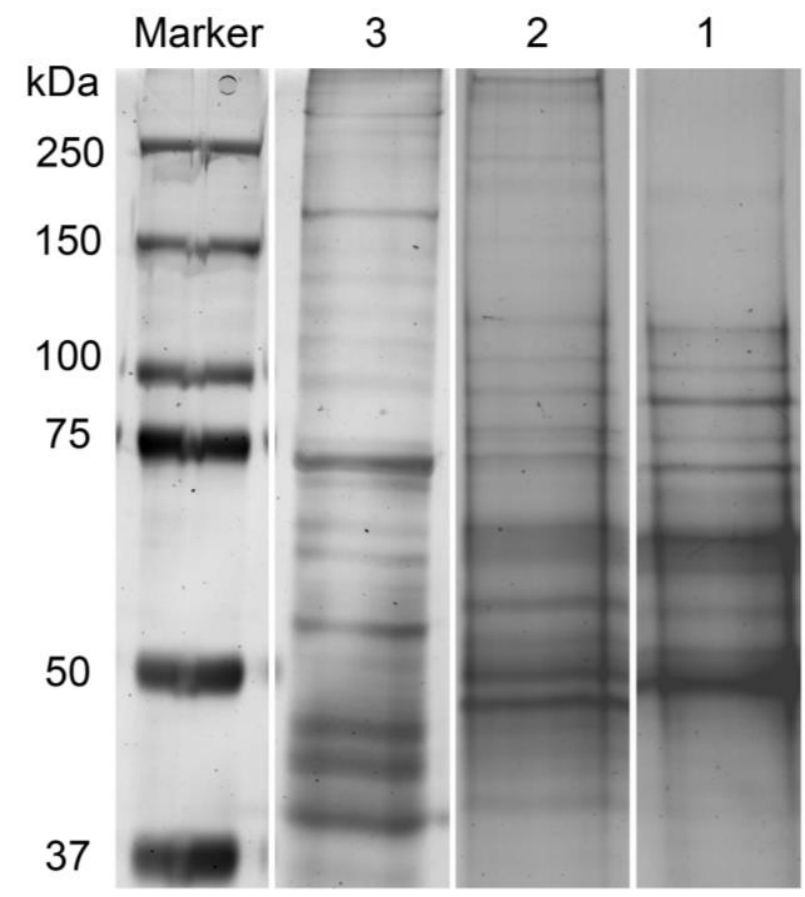

Supplementary Figure S9. Silver stained of SDS-PAGE gel was used to visualize intracellular and extracellular exchange of serum corona on AuNPs. Lane 1 showed the corona proteins around AuNPs incubated in serum for $1 \mathrm{~h}$; Lane 2 showed the corona proteins around AuNPs that preincubated in serum, followed by incubation in cell lysate from HepG2 cells for $1 \mathrm{~h}$; Lane 3 showed the corona proteins around AuNPs recovered from HepG2 cells with PFA cross-linking prior to cell lysis process. The protein samples in lanes 1 and 2 were washed by RIPA as in lane 3, but did not undergo PFA cross-linking. 


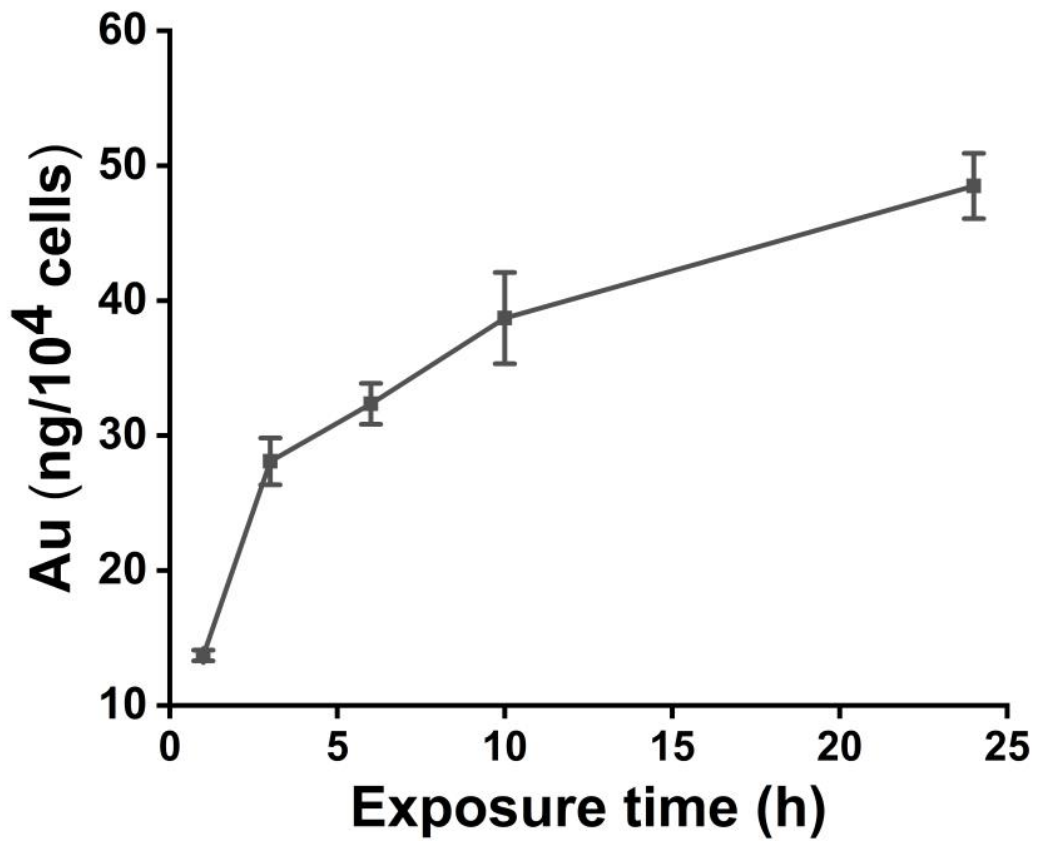

Supplementary Figure S10. Cellular uptake of $20 \mu \mathrm{g} / \mathrm{mL}$ AuNPs at different incubated time in HepG2 cells was measured by ICP-MS. The results showed a continuous uptake of AuNPs (20 mg/L) over $24 \mathrm{~h}$. 
(a)

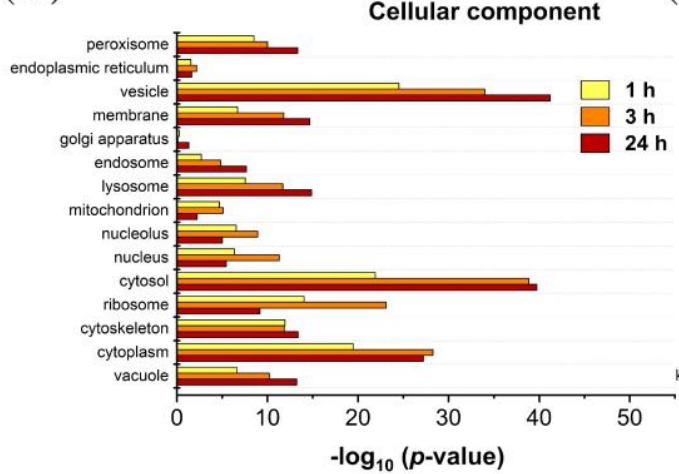

(b)

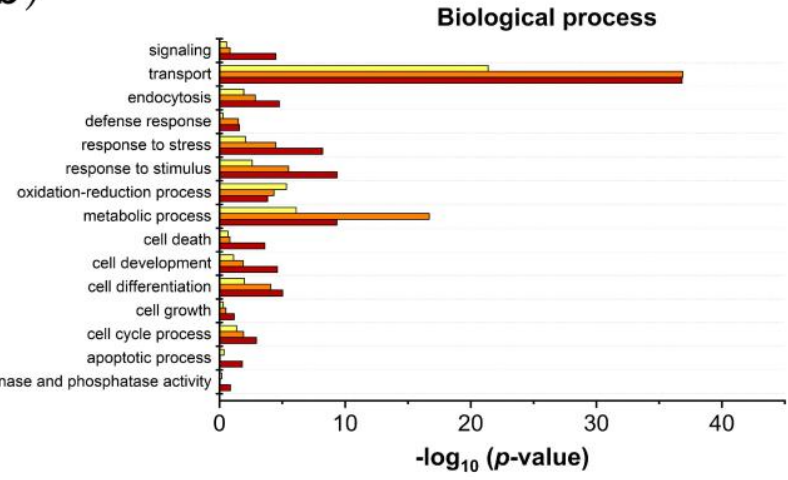

(c)

\begin{tabular}{|c|c|c|c|c|c|c|c|}
\hline \multirow{2}{*}{ Cellular component terms } & \multicolumn{3}{|c|}{$p$-value } & \multirow{2}{*}{ Biological process terms } & \multicolumn{3}{|c|}{$p$-value } \\
\hline & $1 \mathrm{~h}$ & $3 \mathrm{~h}$ & $24 \mathrm{~h}$ & & $1 \mathrm{~h}$ & $3 \mathrm{~h}$ & $24 \mathrm{~h}$ \\
\hline vesicle & $3.08 \mathrm{E}-25$ & $9.46 \mathrm{E}-35$ & $5.93 \mathrm{E}-42$ & transport & 4. $16 \mathrm{E}-22$ & $1.33 \mathrm{E}-37$ & $1.51 \mathrm{E}-37$ \\
\hline cytosol & $1.27 \mathrm{E}-22$ & $1.43 \mathrm{E}-39$ & $1.81 \mathrm{E}-40$ & response to stimulus & $2.51 \mathrm{E}-03$ & $3.10 \mathrm{E}-06$ & 4.44E-10 \\
\hline cytoplasm & $3.50 \mathrm{E}-20$ & $5.48 \mathrm{E}-29$ & $6.06 \mathrm{E}-28$ & metabolic process & $7.89 \mathrm{E}-07$ & 2.04E-17 & $4.54 \mathrm{E}-10$ \\
\hline lysosome & $2.92 \mathrm{E}-08$ & $2.03 \mathrm{E}-12$ & $1.39 \mathrm{E}-15$ & response to stress & $8.54 \mathrm{E}-03$ & 3.34E-05 & $6.19 \mathrm{E}-09$ \\
\hline membrane & 2.04E-07 & $1.65 \mathrm{E}-12$ & $2.10 \mathrm{E}-15$ & cell differentiation & $1.04 \mathrm{E}-02$ & $8.14 \mathrm{E}-05$ & 9.44E-06 \\
\hline cytoskeleton & $1.16 \mathrm{E}-12$ & $1.39 \mathrm{E}-12$ & $4.28 \mathrm{E}-14$ & endocytosis & $1.17 \mathrm{E}-02$ & $1.39 \mathrm{E}-03$ & $1.73 \mathrm{E}-05$ \\
\hline peroxisome & $3.15 \mathrm{E}-09$ & $9.75 \mathrm{E}-11$ & $4.60 \mathrm{E}-14$ & cell development & 7.52E-02 & $1.27 \mathrm{E}-02$ & 2.42E-05 \\
\hline vacuole & $2.59 \mathrm{E}-07$ & $6.33 \mathrm{E}-11$ & $5.94 \mathrm{E}-14$ & signaling & $2.78 \mathrm{E}-01$ & $1.46 \mathrm{E}-01$ & 3.26E-05 \\
\hline ribosome & $9.10 \mathrm{E}-15$ & $7.73 \mathrm{E}-24$ & $7.14 \mathrm{E}-10$ & oxidation-reduction process & 4.73E-06 & 4.62E-05 & $1.49 \mathrm{E}-04$ \\
\hline endosome & $2.04 \mathrm{E}-03$ & $1.45 \mathrm{E}-05$ & $2.10 \mathrm{E}-08$ & cell death & $2.05 \mathrm{E}-01$ & $1.49 \mathrm{E}-01$ & $2.43 \mathrm{E}-04$ \\
\hline nucleus & 4.57E-07 & $4.96 \mathrm{E}-12$ & $3.56 \mathrm{E}-06$ & cell cycle process & $4.31 \mathrm{E}-02$ & $1.23 \mathrm{E}-02$ & $1.14 \mathrm{E}-03$ \\
\hline nucleolus & $2.84 \mathrm{E}-07$ & $1.22 \mathrm{E}-09$ & $9.84 \mathrm{E}-06$ & apoptotic process & 4.32E-01 & $8.76 \mathrm{E}-01$ & $1.51 \mathrm{E}-02$ \\
\hline mitochondrion & 2.12E-05 & 7.96E-06 & $5.88 \mathrm{E}-03$ & defense response & $5.26 \mathrm{E}-01$ & $3.13 \mathrm{E}-02$ & $2.56 \mathrm{E}-02$ \\
\hline endoplasmic reticulum & $2.90 \mathrm{E}-02$ & $6.57 \mathrm{E}-03$ & $2.31 \mathrm{E}-02$ & cell growth & $5.07 \mathrm{E}-01$ & 3.03E-01 & $6.69 \mathrm{E}-02$ \\
\hline golgi apparatus & $5.53 \mathrm{E}-01$ & $8.11 \mathrm{E}-01$ & $4.86 \mathrm{E}-02$ & kinase and phosphatase activity & $6.68 \mathrm{E}-01$ & $8.65 \mathrm{E}-01$ & $1.32 \mathrm{E}-01$ \\
\hline
\end{tabular}

Supplementary Figure S11. Gene ontology (GO) analysis of the identified proteins with an enrichment factor $>2$-fold. (a) cellular component (b) biological process. $X$-axis is the adjusted $p$-value transformed by $-\log _{10}$, and the $y$-axis represents the GO functional classification. (c) Specific $p$-value for each category. 


\begin{tabular}{|c|c|c|}
\hline Pretreatment & Concentration & Inhibited Pathway \\
\hline $4{ }^{\circ} \mathrm{C}$ & N.A. & energy-dependent endocytosis \\
\hline chlorpromazine & $10 \mu \mathrm{g} / \mathrm{mL}$ & clathrin-mediated endocytosis \\
\hline dynasore & $100 \mu \mathrm{M}$ & dynamin-dependent endocytosis \\
\hline EIPA & $100 \mu \mathrm{M}$ & macropinocytosis \\
\hline methyl- $\beta$-cyclodextrin & $2.5 \mathrm{mg} / \mathrm{mL}$ & $\begin{array}{l}\text { lipid-raft/caveolae-, clathrin-mediated } \\
\text { endocytosis and fluid-phase endocytosis }\end{array}$ \\
\hline polyinosinic acid & $25 \mu \mathrm{g} / \mathrm{mL}$ & scavenger receptor-mediated endocytosis \\
\hline
\end{tabular}

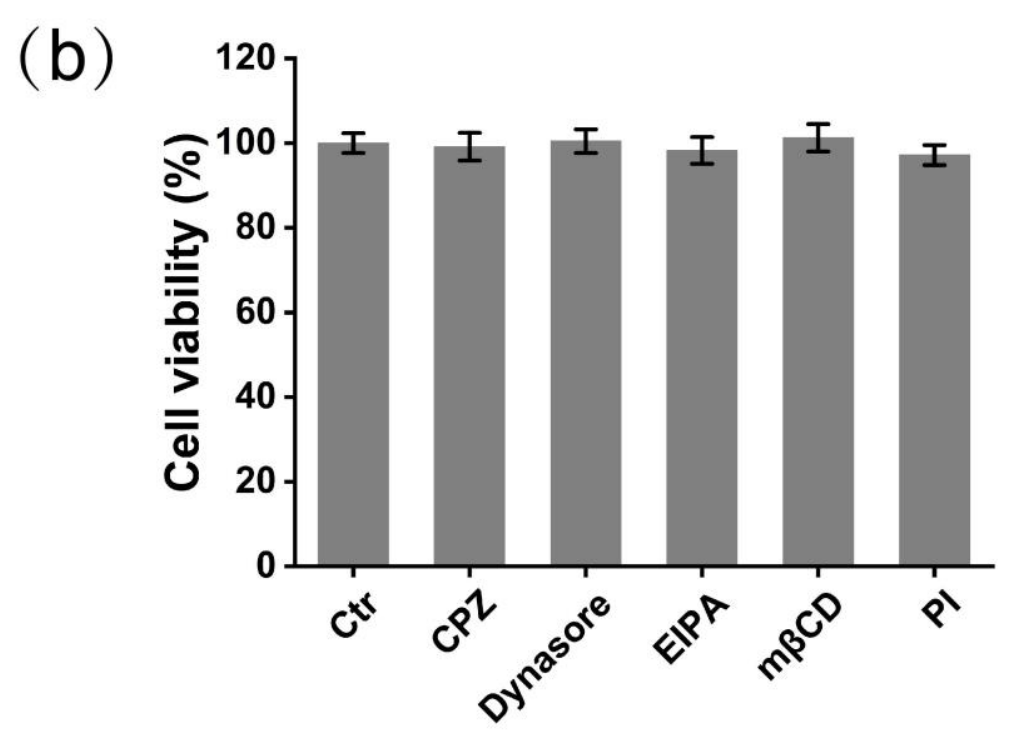

Supplementary Figure S12. (a) The table displays the pharmacological inhibitor used in the present study, together with their applied concentrations and blocked endocytic pathway. (b) The effects of different pharmacological inhibitor on HepG2 cell viability were measured by MTT assay. The results show that the cells treated with these inhibitors for $3 \mathrm{~h}$ kept largely viable. Values are mean $\pm \mathrm{SD}$ from at least three independent experiments. 


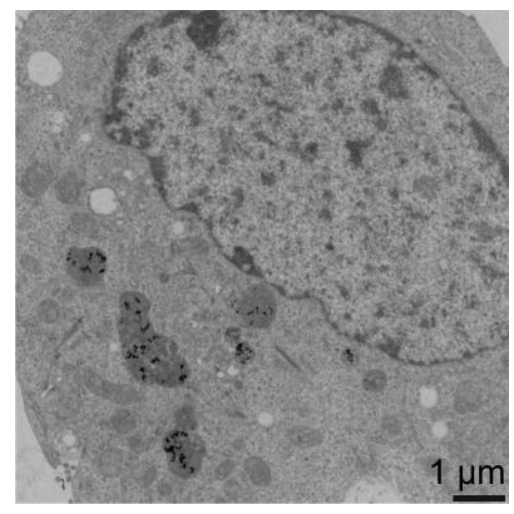

Supplementary Figure S13. TEM image showing that most of internalized AuNPs were detected in lysosomes in HepG2 cells after 24 of incubation. 
Supplementary Table S1. Physicochemical properties of the AuNPs in the absence and presence of non-cross-linked and cross-linked corona ${ }^{a}$.

\begin{tabular}{cccccc} 
& Bare & \multicolumn{2}{c}{ LC } & \multicolumn{2}{c}{ HC } \\
\cline { 3 - 6 } & & Non-cross-linking & Cross-linking & Non-cross-linking & Cross-linking \\
$d_{\mathrm{h}, \mathrm{n}}(\mathrm{nm})$ & $13.73 \pm 0.40$ & $30.37 \pm 0.06$ & $25.34 \pm 1.18$ & $33.91 \pm 1.25$ & $26.10 \pm 0.41$ \\
$\lambda \max (\mathrm{nm})$ & 520 & 525 & 523 & 527 & 525 \\
$\zeta$ app $(\mathrm{mV})$ & $-30.77 \pm 0.99$ & $-12.83 \pm 0.38$ & $-10.47 \pm 0.39$ & $-14.66 \pm 0.95$ & $-12.53 \pm 1.08$ \\
\hline
\end{tabular}

${ }^{a}$ Abbreviations: $d_{\mathrm{h}, \mathrm{n}}$, number mean hydrodynamic diameter; $\lambda$ max, plasmon absorbance wavelength maximum; $\zeta^{\text {app }}$, apparent zeta potential; LC, low-serum corona coated AuNPs; HC, high-serum corona coated AuNPs. 
Supplementary Table S2. Recovery of nanoparticle after centrifugation. The AuNPprotein complexes were formed in serum and collected as described in the Methods. The content of AuNPs recovered in the supernatant and in the pellet for samples with or without cross-linking were quantified by ICP-MS. Briefly, after centrifugation, the supernatant containing unbound serum and the resuspended pellet were digested in aqua regia for overnight to dissolve AuNPs. After the solution was evaporated and diluted with $2 \% \mathrm{HNO}_{3}$, the amount of $\mathrm{Au}$ was determined by ICP-MS. The results are expressed as a percentage relative to the content of the initial AuNPs dispersion before centrifugation. More than $94 \%$ of the nanoparticles could be recovered at our current centrifugation speed (more than $87 \%$ even after 3 centrifugations). Besides, the amounts of AuNPs recovered for cross-linked and non-cross-linked samples were comparable, indicating that their differences in protein patterns (see Figure 4 in the main manuscript) are not a consequence of nanoparticle loss in the isolation procedure.

\begin{tabular}{ccccc} 
& \multicolumn{2}{c}{ PBS } & \multicolumn{2}{c}{ RIPA } \\
\cline { 2 - 5 } & Non-cross-linking & Cross-linking & Non-cross-linking & Cross-linking \\
Supernatant & $4.0 \pm 0.8 \%$ & $5.0 \pm 0.2 \%$ & $8.0 \pm 0.6 \%$ & $6 \pm 0.7 \%$ \\
Pellet (1st centrifugation) & $97 \pm 3.1 \%$ & $98 \pm 3.8 \%$ & $95 \pm 1.5 \%$ & $94 \pm 2.1 \%$ \\
Pellet (3th centrifugation) & $91 \pm 2.2 \%$ & $90 \pm 3.5 \%$ & $89 \pm 2.6 \%$ & $87 \pm 1.4 \%$ \\
\hline
\end{tabular}

PBS: the complexes were washed with PBS. RIPA: the complexes were washed with RIPA. 
Supplementary Table S6. Top 50 most-abundant proteins enriched in the corona of AuNPs at different time of cells exposure.

\begin{tabular}{|c|c|c|c|}
\hline \multirow{2}{*}{ Protein } & \multicolumn{3}{|c|}{ Composition of corona $(\%)$} \\
\hline & $1 \mathrm{~h}$ & $3 \mathrm{~h}$ & $24 \mathrm{~h}$ \\
\hline 1 Actin, cytoplasmic 2 & 12.754 & 10.303 & 16.151 \\
\hline 2 Peroxisomal multifunctional enzyme type 2 & 1.072 & 2.835 & 5.703 \\
\hline 3 Clathrin heavy chain 1 & 1.184 & 1.694 & 3.362 \\
\hline 4 Catalase & 0.278 & 0.346 & 3.125 \\
\hline 5 Spectrin beta chain, non-erythrocytic 1 & 2.287 & 2.762 & 3.049 \\
\hline 6 Myosin-9 & 11.698 & 11.452 & 2.614 \\
\hline 7 Spectrin alpha chain, non-erythrocytic 1 & 2.565 & 2.621 & 2.375 \\
\hline 8 Heat shock protein HSP 90-beta & 1.414 & 1.424 & 2.278 \\
\hline 9 Myosin-10 & 8.002 & 6.785 & 2.239 \\
\hline 10 Filamin-B & 0.943 & 0.798 & 1.877 \\
\hline 11 Fatty acid synthase & 0.932 & 0.975 & 1.797 \\
\hline 12 Stress-70 protein, mitochondrial & 1.291 & 1.432 & 1.711 \\
\hline 13 Filamin-A & 0.731 & 0.870 & 1.706 \\
\hline 14 Peroxisomal bifunctional enzyme & 0.209 & 0.662 & 1.689 \\
\hline 15 Histone $\mathrm{H} 4$ & 1.493 & 1.237 & 1.581 \\
\hline 16 Peroxisomal acyl-coenzyme A oxidase 1 & 0.293 & 0.421 & 1.327 \\
\hline 17 Histone $\mathrm{H} 3.2$ & 0.875 & 0.577 & 1.087 \\
\hline 18 Putative elongation factor 1 -alpha-like 3 & 0.624 & 0.586 & 0.988 \\
\hline 19 Heat shock cognate $71 \mathrm{kDa}$ protein & 0.402 & 0.581 & 0.936 \\
\hline 20 Dipeptidyl peptidase 4 & 0.238 & 0.124 & 0.871 \\
\hline 21 Elongation factor 2 & 0.564 & 0.770 & 0.844 \\
\hline 22 Apolipoprotein B-100 & $*$ & 0.092 & 0.805 \\
\hline 23 Enoyl-CoA delta isomerase 2, mitochondrial & 0.094 & 0.230 & 0.795 \\
\hline 24 Alpha-actinin-1 & 0.383 & 0.506 & 0.786 \\
\hline 25 Tubulin beta-4B chain & 0.659 & 0.448 & 0.783 \\
\hline 26 Histone H2A type $1-\mathrm{J}$ & 1.005 & 0.371 & 0.771 \\
\hline 27 Alkyldihydroxyacetonephosphate synthase, peroxisomal & 0.139 & 0.321 & 0.712 \\
\hline 28 Actin, alpha skeletal muscle & 0.643 & 0.867 & 0.708 \\
\hline 29 Peroxisomal sarcosine oxidase & 0.043 & 0.099 & 0.707 \\
\hline 30 Alpha-actinin-4 & 0.294 & 0.373 & 0.668 \\
\hline 31 3-ketoacyl-CoA thiolase, peroxisomal & 0.118 & 0.232 & 0.604 \\
\hline 32 Delta-1-pyrroline-5-carboxylate synthase & 13.975 & 15.268 & 0.582 \\
\hline 33 Histone $\mathrm{H} 2 \mathrm{~B}$ type $1-\mathrm{L}$ & 0.533 & 0.421 & 0.570 \\
\hline 34 Desmoplakin & 2.772 & 2.404 & 0.513 \\
\hline 35 Non-specific lipid-transfer protein & 0.108 & 0.119 & 0.428 \\
\hline 36 Serine--pyruvate aminotransferase & 0.000 & 0.000 & 0.398 \\
\hline 37 Aminopeptidase $\mathrm{N}$ & 0.266 & 0.160 & 0.330 \\
\hline 38 Ubiquitin-40S ribosomal protein S27a & 0.196 & 0.181 & 0.330 \\
\hline 39 Peroxisomal trans-2-enoyl-CoA reductase & 0.064 & 0.090 & 0.327 \\
\hline 40 Ras GTPase-activating-like protein IQGAP2 & $*$ & 0.075 & 0.312 \\
\hline
\end{tabular}


41 Heat shock $70 \mathrm{kDa}$ protein 1B

$\begin{array}{ccc}0.113 & 0.148 & 0.298 \\ 0.202 & 0.281 & 0.294 \\ 0.054 & 0.042 & 0.292 \\ 0.092 & 0.075 & 0.288 \\ 0.589 & 0.705 & 0.282 \\ 0.000 & 0.074 & 0.273 \\ 0.102 & 0.134 & 0.256 \\ 0.726 & 0.545 & 0.224 \\ 0.046 & 0.056 & 0.211 \\ * & * & 0.199\end{array}$

42 Endoplasmin

43 Radixin

44 AP-2 complex subunit beta

45 Junction plakoglobin

46 Dihydroxyacetone phosphate acyltransferase

47 Long-chain-fatty-acid--CoA ligase 4

48 40S ribosomal protein S8

49 Ras GTPase-activating-like protein IQGAP1

50 Peroxisomal acyl-coenzyme A oxidase 2

* indicates that the protein was undetectable. 
Supplementary Table S7. The identified proteins with an enrichment factor $>2$-fold belonging to the endocytosis pathway at different time of cells exposure.

\begin{tabular}{|c|c|c|c|}
\hline $1 \mathrm{~h}$ & Ref number & Description & Gene Symbol \\
\hline & O95782 & AP-2 complex subunit alpha-1 & AP2A1 \\
\hline & O94973 & AP-2 complex subunit alpha-2 & AP2A2 \\
\hline & P63010 & AP-2 complex subunit beta & $\mathrm{AP} 2 \mathrm{~B} 1$ \\
\hline & Q00610 & Clathrin heavy chain 1 & CLTC \\
\hline & Q9H4M9 & EH domain-containing protein 1 & EHD1 \\
\hline & Q9Y6I3 & Epsin-1 & EPN1 \\
\hline & P14625 & Endoplasmin & HSP90B1 \\
\hline & P35579 & Myosin-9 & MYH9 \\
\hline & P51148 & Ras-related protein $\mathrm{Rab}-5 \mathrm{C}$ & RAB5C \\
\hline & Q99961 & Endophilin-A2 & SH3GL1 \\
\hline
\end{tabular}

\begin{tabular}{ccc} 
Ref number & Description & Gene Symbol \\
O95782 & AP-2 complex subunit alpha-1 & AP2A1 \\
O94973 & AP-2 complex subunit alpha-2 & AP2A2 \\
P63010 & AP-2 complex subunit beta & AP2B1 \\
Q96CW1 & AP-2 complex subunit mu & AP2M1 \\
P04114 & Apolipoprotein B-100 & APOB \\
Q00610 & Clathrin heavy chain 1 & CLTC \\
P50570 & Dynamin-2 & DNM2 \\
Q9H4M9 & EH domain-containing protein 1 & EHD1 \\
Q9Y6I3 & Epsin-1 & EPN1 \\
P15311 & Ezrin & EZR \\
P14625 & Endoplasmin & HSP90B1 \\
P01876 & Immunoglobulin heavy constant alpha 1 & IGHA1 \\
P35579 & Myosin-9 & MYH9 \\
P51148 & Ras-related protein Rab-5C & RAB5C \\
P51149 & Ras-related protein Rab-7a & RAB7A \\
Q99961 & Endophilin-A2 & SH3GL1 \\
\hline
\end{tabular}

$\begin{array}{ccc}24 \mathrm{~h} & \text { Def number } & \text { Gene Symbol } \\ \text { P04114 } & \text { Apolipoprotein B-100 } & \text { APOB } \\ \text { Q00610 } & \text { Clathrin heavy chain 1 } & \text { CLTC } \\ \text { Q96CW1 } & \text { AP-2 complex subunit mu } & \text { AP2M1 } \\ \text { Q9Y5X3 } & \text { Sorting nexin-5 } & \text { SNX5 } \\ \text { P09496 } & \text { Clathrin light chain A } & \text { CLTA } \\ \text { O95782 } & \text { AP-2 complex subunit alpha-1 } & \text { AP2A1 } \\ \text { Q99961 } & \text { Endophilin-A2 } & \text { SH3GL1 } \\ \text { P63010 } & \text { AP-2 complex subunit beta } & \text { AP2B1 } \\ \text { P35579 } & \text { Myosin-9 } & \text { MYH9 } \\ \text { P15311 } & \text { Ezrin } & \text { EZR } \\ & & \end{array}$


O94973

P61966

P14625

P51148

P51149

Q14247

P50570

Q9Y6I3
AP-2 complex subunit alpha-2

AP2A2

AP-1 complex subunit sigma-1A

Endoplasmin

Ras-related protein Rab-5C

Ras-related protein Rab-7a

Src substrate cortactin

Dynamin-2

Epsin-1
AP1S1

HSP90B 1

RAB5C

RAB7A

CTTN

DNM2

EPN1 
Supplementary Table S8. The identified proteins with an enrichment factor $>2$-fold belonging to the vesicle-mediated transport pathway at different time of cells exposure.

\begin{tabular}{|c|c|c|c|}
\hline $1 \mathrm{~h}$ & $\begin{array}{c}\text { Ref } \\
\text { number }\end{array}$ & Description & $\begin{array}{c}\text { Gene } \\
\text { Symbol }\end{array}$ \\
\hline & P09110 & 3-ketoacyl-CoA thiolase, peroxisomal & ACAA1 \\
\hline & P63261 & Actin, cytoplasmic 2 & ACTG1 \\
\hline & P12814 & Alpha-actinin-1 & ACTN1 \\
\hline & O43707 & Alpha-actinin-4 & ACTN4 \\
\hline & P61160 & Actin-related protein 2 & ACTR2 \\
\hline & P15144 & Aminopeptidase $\mathrm{N}$ & ANPEP \\
\hline & O95782 & AP-2 complex subunit alpha-1 & AP2A1 \\
\hline & O94973 & AP-2 complex subunit alpha-2 & AP2A2 \\
\hline & P63010 & AP-2 complex subunit beta & AP2B1 \\
\hline & O15143 & Actin-related protein $2 / 3$ complex subunit $1 \mathrm{~B}$ & ARPC1B \\
\hline & P04040 & Catalase & CAT \\
\hline & P50990 & T-complex protein 1 subunit theta & СCT8 \\
\hline & P13987 & CD59 glycoprotein & CD59 \\
\hline & Q00610 & Clathrin heavy chain 1 & CLTC \\
\hline & P53621 & Coatomer subunit alpha & COPA \\
\hline & Q9Y678 & Coatomer subunit gamma-1 & COPG1 \\
\hline & Q9ULV4 & Coronin-1C & CORO1C \\
\hline & P15924 & Desmoplakin & DSP \\
\hline & Q14204 & Cytoplasmic dynein 1 heavy chain 1 & DYNC1H1 \\
\hline & Q9H4M9 & EH domain-containing protein 1 & EHD1 \\
\hline & Q9Y6I3 & Epsin-1 & EPN1 \\
\hline & $\mathrm{P} 21333$ & Filamin-A & FLNA \\
\hline & P02794 & Ferritin heavy chain & FTH1 \\
\hline & P02792 & Ferritin light chain & FTL \\
\hline & P14625 & Endoplasmin & HSP90B 1 \\
\hline & P17066 & Heat shock $70 \mathrm{kDa}$ protein 6 & HSPA6 \\
\hline & P11142 & Heat shock cognate $71 \mathrm{kDa}$ protein & HSPA8 \\
\hline & P14923 & Junction plakoglobin & JUP \\
\hline & Q02241 & Kinesin-like protein KIF23 & KIF23 \\
\hline & Q14764 & Major vault protein & MVP \\
\hline & P35580 & Myosin-10 & MYH10 \\
\hline & P35579 & Myosin-9 & MYH9 \\
\hline & Q92614 & Unconventional myosin-XVIIIa & MYO18A \\
\hline & O43795 & Unconventional myosin-Ib & MYO1B \\
\hline & Q9H0U4 & Ras-related protein Rab-1B & RAB1B \\
\hline & P51148 & Ras-related protein $\mathrm{Rab}-5 \mathrm{C}$ & RAB5C \\
\hline & Q9H0H5 & Rac GTPase-activating protein 1 & RACGAP1 \\
\hline & P61586 & Transforming protein RhoA & RHOA \\
\hline & P62979 & Ubiquitin-40S ribosomal protein $\mathrm{S} 27 \mathrm{a}$ & RPS27A \\
\hline & Q15436 & Protein transport protein Sec23A & SEC23A \\
\hline
\end{tabular}




\begin{tabular}{ccc} 
Q99961 & Endophilin-A2 & SH3GL1 \\
Q13813 & Spectrin alpha chain, non-erythrocytic 1 & SPTAN1 \\
Q01082 & Spectrin beta chain, non-erythrocytic 1 & SPTBN1 \\
Q13501 & Sequestosome-1 & SQSTM1 \\
P27105 & Erythrocyte band 7 integral membrane protein & STOM \\
O15260 & Surfeit locus protein 4 & SURF4 \\
Q9Y490 & Talin-1 & TLN1 \\
P07437 & Tubulin beta chain & TUBB \\
P68371 & Tubulin beta-4B chain & TUBB4B \\
P18206 & Vinculin & VCL \\
Q96QK1 & Vacuolar protein sorting-associated protein 35 & VPS35 \\
\hline
\end{tabular}

$\begin{array}{ccc}\text { 3h h } & & \text { Gene } \\ \text { Ref number } & \text { Description } & \text { Symbol } \\ \text { P09110 } & \text { 3-ketoacyl-CoA thiolase, peroxisomal } & \text { ACAA1 } \\ \text { P63261 } & \text { Actin, cytoplasmic 2 } & \text { ACTG1 } \\ \text { P12814 } & \text { Alpha-actinin-1 } & \text { ACTN1 } \\ \text { O43707 } & \text { Alpha-actinin-4 } & \text { ACTN4 } \\ \text { P61160 } & \text { Actin-related protein 2 } & \text { ACTR2 } \\ \text { P15144 } & \text { Aminopeptidase N } & \text { ANPEP } \\ \text { P07355 } & \text { Annexin A2 } & \text { ANXA2 } \\ \text { O95782 } & \text { AP-2 complex subunit alpha-1 } & \text { AP2A1 } \\ \text { O94973 } & \text { AP-2 complex subunit alpha-2 } & \text { AP2A2 } \\ \text { P63010 } & \text { AP-2 complex subunit beta } & \text { AP2B1 } \\ \text { Q96CW1 } & \text { AP-2 complex subunit mu } & \text { AP2M1 } \\ \text { O00203 } & \text { AP-3 complex subunit beta-1 } & \text { AP3B1 } \\ \text { P04114 } & \text { Apolipoprotein B-100 } & \text { APOB } \\ \text { P05089 } & \text { Arginase-1 } & \text { ARG1 } \\ \text { O15143 } & \text { Actin-related protein 2/3 complex subunit 1B } & \text { ARPC1B } \\ \text { O15144 } & \text { Actin-related protein 2/3 complex subunit 2 } & \text { ARPC2 } \\ \text { P59998 } & \text { Actin-related protein 2/3 complex subunit 4 } & \text { ARPC4 } \\ \text { Q86VP6 } & \text { Cullin-associated NEDD8-dissociated protein 1 } & \text { CAND1 } \\ \text { P47756 } & \text { F-actin-capping protein subunit beta } & \text { CAPZB } \\ \text { P04040 } & \text { Catalase } & \text { CAT } \\ \text { P50990 } & \text { T-complex protein 1 subunit theta } & \text { CCT8 } \\ \text { Q07065 } & \text { Cytoskeleton-associated protein 4 } & \text { CKAP4 } \\ \text { Q00610 } & \text { Clathrin heavy chain 1 } & \text { CLTC } \\ \text { P53621 } & \text { Coatomer subunit alpha } & \text { COPA } \\ \text { P35606 } & \text { Coatomer subunit beta' } & \text { COPB2 } \\ \text { Q9ULV4 } & \text { Coronin-1C } & \text { CORO1C } \\ \text { P50570 } & \text { Dynamin-2 } & \text { DNM2 } \\ \text { Q08554 } & \text { Desmocollin-1 } & \text { DSC1 } \\ \text { Q02413 } & \text { Desmoglein-1 } & \text { DSG1 } \\ \text { P15924 } & \text { DSP } \\ & & \end{array}$




\begin{tabular}{|c|c|c|}
\hline Q14204 & Cytoplasmic dynein 1 heavy chain 1 & DYNC1H1 \\
\hline P13639 & Elongation factor 2 & EEF2 \\
\hline Q9H4M9 & EH domain-containing protein 1 & EHD1 \\
\hline Q9Y6I3 & Epsin-1 & EPN1 \\
\hline P15311 & Ezrin & EZR \\
\hline P21333 & Filamin-A & FLNA \\
\hline P02751 & Fibronectin & FN1 \\
\hline P02794 & Ferritin heavy chain & FTH1 \\
\hline P02792 & Ferritin light chain & FTL \\
\hline P08238 & Heat shock protein HSP 90-beta & HSP90AB \\
\hline P14625 & Endoplasmin & HSP90B1 \\
\hline P0DMV9 & Heat shock $70 \mathrm{kDa}$ protein $1 \mathrm{~B}$ & HSPA1B \\
\hline P17066 & Heat shock $70 \mathrm{kDa}$ protein 6 & HSPA6 \\
\hline P11142 & Heat shock cognate $71 \mathrm{kDa}$ protein & HSPA8 \\
\hline P01876 & Immunoglobulin heavy constant alpha 1 & IGHA1 \\
\hline Q13576 & Ras GTPase-activating-like protein IQGAP2 & IQGAP2 \\
\hline P14923 & Junction plakoglobin & JUP \\
\hline Q02241 & Kinesin-like protein KIF23 & KIF23 \\
\hline Q14764 & Major vault protein & MVP \\
\hline P35580 & Myosin-10 & MYH10 \\
\hline P35579 & Myosin-9 & MYH9 \\
\hline Q92614 & Unconventional myosin-XVIIIa & MYO18A \\
\hline O43795 & Unconventional myosin-Ib & MYO1B \\
\hline O00159 & Unconventional myosin-Ic & MYO1C \\
\hline Q9UQ80 & Proliferation-associated protein $2 \mathrm{G} 4$ & PA2G4 \\
\hline P17858 & ATP-dependent 6-phosphofructokinase, liver type & PFKL \\
\hline P28066 & Proteasome subunit alpha type- 5 & PSMA5 \\
\hline P62491 & Ras-related protein Rab-11A & RAB11A \\
\hline Q9H0U4 & Ras-related protein Rab-1B & RAB1B \\
\hline P51148 & Ras-related protein $\mathrm{Rab}-5 \mathrm{C}$ & RAB5C \\
\hline P51149 & Ras-related protein Rab-7a & RAB7A \\
\hline Q9H0H5 & Rac GTPase-activating protein 1 & RACGAP1 \\
\hline P62979 & Ubiquitin-40S ribosomal protein $\mathrm{S} 27 \mathrm{a}$ & RPS27A \\
\hline P31151 & Protein S100-A7 & S100A7 \\
\hline Q9NR31 & GTP-binding protein SAR1a & SAR1A \\
\hline P53992 & Protein transport protein Sec $24 \mathrm{C}$ & SEC 24C \\
\hline Q99961 & Endophilin-A2 & SH3GL1 \\
\hline Q13813 & Spectrin alpha chain, non-erythrocytic 1 & SPTAN1 \\
\hline Q01082 & Spectrin beta chain, non-erythrocytic 1 & SPTBN1 \\
\hline Q13501 & Sequestosome-1 & SQSTM1 \\
\hline Q92783 & Signal transducing adapter molecule 1 & STAM \\
\hline P27105 & Erythrocyte band 7 integral membrane protein & STOM \\
\hline $\mathrm{O} 15260$ & Surfeit locus protein 4 & SURF4 \\
\hline Q9Y490 & Talin-1 & TLN1 \\
\hline
\end{tabular}


P14373

P07437

P68371

P18206

Q96QK1

Q9UN37
Zinc finger protein RFP

Tubulin beta chain

Tubulin beta-4B chain

Vinculin
TRIM27

TUBB

TUBB4B

VCL

VPS35

VPS4A

\begin{tabular}{|c|c|c|c|}
\hline $24 \mathrm{~h}$ & Ref number & Description & Gene Symbol \\
\hline & P09110 & 3-ketoacyl-CoA thiolase, peroxisomal & ACAA1 \\
\hline & P63261 & Actin, cytoplasmic 2 & ACTG1 \\
\hline & P12814 & Alpha-actinin-1 & ACTN1 \\
\hline & O43707 & Alpha-actinin-4 & ACTN4 \\
\hline & P42025 & Beta-centractin & ACTR1B \\
\hline & P61160 & Actin-related protein 2 & ACTR2 \\
\hline & P61158 & Actin-related protein 3 & ACTR3 \\
\hline & P15144 & Aminopeptidase $\mathrm{N}$ & ANPEP \\
\hline & P07355 & Annexin A2 & ANXA2 \\
\hline & P61966 & AP-1 complex subunit sigma-1A & AP1S1 \\
\hline & O95782 & AP-2 complex subunit alpha-1 & AP2A1 \\
\hline & O94973 & AP-2 complex subunit alpha- 2 & $\mathrm{AP} 2 \mathrm{~A} 2$ \\
\hline & P63010 & AP-2 complex subunit beta & AP2B1 \\
\hline & Q96CW1 & AP-2 complex subunit mu & AP2M1 \\
\hline & P04114 & Apolipoprotein B-100 & APOB \\
\hline & P05089 & Arginase-1 & ARG1 \\
\hline & O15143 & Actin-related protein $2 / 3$ complex subunit 1B & ARPC1B \\
\hline & Q93050 & V-type proton ATPase $116 \mathrm{kDa}$ subunit a isoform 1 & ATP6V0A1 \\
\hline & P27449 & V-type proton ATPase $16 \mathrm{kDa}$ proteolipid subunit & ATP6V0C \\
\hline & P47756 & F-actin-capping protein subunit beta & CAPZB \\
\hline & P04040 & Catalase & CAT \\
\hline & P50990 & T-complex protein 1 subunit theta & ССТ8 \\
\hline & Q9Y592 & Centrosomal protein of $83 \mathrm{kDa}$ & CEP83 \\
\hline & Q9H444 & Charged multivesicular body protein $4 \mathrm{~b}$ & CHMP4B \\
\hline & P09496 & Clathrin light chain A & CLTA \\
\hline & Q00610 & Clathrin heavy chain 1 & CLTC \\
\hline & P53621 & Coatomer subunit alpha & COPA \\
\hline & P35606 & Coatomer subunit beta' & COPB2 \\
\hline & Q9ULV4 & Coronin-1C & CORO1C \\
\hline & Q14247 & Src substrate cortactin & CTTN \\
\hline & P50570 & Dynamin-2 & DNM2 \\
\hline & Q02413 & Desmoglein-1 & DSG1 \\
\hline & P15924 & Desmoplakin & DSP \\
\hline & P13639 & Elongation factor 2 & EEF2 \\
\hline & Q9Y6I3 & Epsin-1 & EPN1 \\
\hline & P15311 & Ezrin & EZR \\
\hline & & S24 & \\
\hline
\end{tabular}




\begin{tabular}{|c|c|c|}
\hline P21333 & Filamin-A & FLNA \\
\hline P02751 & Fibronectin & FN1 \\
\hline P02794 & Ferritin heavy chain & FTH1 \\
\hline P02792 & Ferritin light chain & FTL \\
\hline P08238 & Heat shock protein HSP 90-beta & HSP90AB1 \\
\hline P14625 & Endoplasmin & HSP90B 1 \\
\hline P0DMV9 & Heat shock $70 \mathrm{kDa}$ protein $1 \mathrm{~B}$ & HSPA1B \\
\hline P17066 & Heat shock $70 \mathrm{kDa}$ protein 6 & HSPA6 \\
\hline P11142 & Heat shock cognate $71 \mathrm{kDa}$ protein & HSPA8 \\
\hline P46940 & Ras GTPase-activating-like protein IQGAP1 & IQGAP1 \\
\hline Q13576 & Ras GTPase-activating-like protein IQGAP2 & IQGAP2 \\
\hline P14923 & Junction plakoglobin & JUP \\
\hline Q02241 & Kinesin-like protein KIF23 & KIF23 \\
\hline P13473 & Lysosome-associated membrane glycoprotein 2 & LAMP2 \\
\hline P35580 & Myosin-10 & MYH10 \\
\hline P35579 & Myosin-9 & MYH9 \\
\hline Q92614 & Unconventional myosin-XVIIIa & MYO18A \\
\hline O43795 & Unconventional myosin-Ib & MYO1B \\
\hline O00159 & Unconventional myosin-Ic & MYO1C \\
\hline P17858 & ATP-dependent 6-phosphofructokinase, liver type & PFKL \\
\hline P62937 & Peptidyl-prolyl cis-trans isomerase A & PPIA \\
\hline P28066 & Proteasome subunit alpha type-5 & PSMA5 \\
\hline P62491 & Ras-related protein Rab-11A & RAB11A \\
\hline Q9H0U4 & Ras-related protein Rab-1B & RAB1B \\
\hline P51148 & Ras-related protein $\mathrm{Rab}-5 \mathrm{C}$ & RAB5C \\
\hline Q9NRW1 & Ras-related protein Rab-6B & RAB6B \\
\hline P51149 & Ras-related protein Rab-7a & RAB7A \\
\hline Q9H0H5 & Rac GTPase-activating protein 1 & RACGAP1 \\
\hline P61586 & Transforming protein RhoA & RHOA \\
\hline P62979 & Ubiquitin-40S ribosomal protein $\mathrm{S} 27 \mathrm{a}$ & RPS27A \\
\hline Q15436 & Protein transport protein Sec $23 \mathrm{~A}$ & SEC23A \\
\hline Q99961 & Endophilin-A2 & SH3GL1 \\
\hline Q9Y5X3 & Sorting nexin-5 & SNX5 \\
\hline Q13813 & Spectrin alpha chain, non-erythrocytic 1 & SPTAN1 \\
\hline Q01082 & Spectrin beta chain, non-erythrocytic 1 & SPTBN1 \\
\hline Q13501 & Sequestosome-1 & SQSTM1 \\
\hline P27105 & Erythrocyte band 7 integral membrane protein & STOM \\
\hline 015260 & Surfeit locus protein 4 & SURF4 \\
\hline P35625 & Metalloproteinase inhibitor 3 & TIMP3 \\
\hline Q9Y490 & Talin-1 & TLN1 \\
\hline P07437 & Tubulin beta chain & TUBB \\
\hline P68371 & Tubulin beta-4B chain & TUBB4B \\
\hline Q96QK1 & Vacuolar protein sorting-associated protein 35 & VPS35 \\
\hline
\end{tabular}

